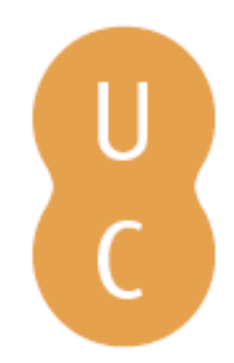

\title{
pommalina
}

\section{Comment combler les obstacles linguistico-culturels dans la traduction d'un mets vietnamien?}

\author{
Autor(es): $\quad$ Lo-Cicero, Minh $\mathrm{Ha}$ \\ Publicado por: Imprensa da Universidade de Coimbra \\ URL \\ persistente: URI:http://hdl.handle.net/10316.2/39643 \\ DOI: $\quad$ DOI:https://doi.org/10.14195/978-989-26-1191-4_14 \\ Accessed : $\quad$ 26-Apr-2023 13:08:58
}

A navegação consulta e descarregamento dos títulos inseridos nas Bibliotecas Digitais UC Digitalis, UC Pombalina e UC Impactum, pressupõem a aceitação plena e sem reservas dos Termos e Condições de Uso destas Bibliotecas Digitais, disponíveis em https://digitalis.uc.pt/pt-pt/termos.

Conforme exposto nos referidos Termos e Condições de Uso, o descarregamento de títulos de acesso restrito requer uma licença válida de autorização devendo o utilizador aceder ao(s) documento(s) a partir de um endereço de IP da instituição detentora da supramencionada licença.

Ao utilizador é apenas permitido o descarregamento para uso pessoal, pelo que o emprego do(s) título(s) descarregado(s) para outro fim, designadamente comercial, carece de autorização do respetivo autor ou editor da obra.

Na medida em que todas as obras da UC Digitalis se encontram protegidas pelo Código do Direito de Autor e Direitos Conexos e demais legislação aplicável, toda a cópia, parcial ou total, deste documento, nos casos em que é legalmente admitida, deverá conter ou fazer-se acompanhar por este aviso.

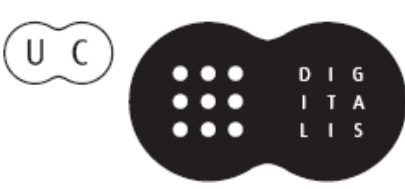




\title{
COMMENT COMBLER LES OBSTACLES LINGUISTICO- CULTURELS DANS LA TRADUCTION D'UN METS VIETNAMIEN? How to bridge linguistic and cultural barriers in the translation of a Vietnamese Dish?
}

\author{
Minh Ha Lo-Cicero \\ Universidade da Madeira \\ mhcicero@staff.uma.pt \\ Faculdade de Artes e Humanidades \\ CLEPUL
}

RÉsumé: La traduction des spécialités ou des recettes vietnamiennes est importante dans la mesure où il y a une communauté très importante de Vietnamiens qui immigrent en France. Partager la culture de l'autre recouvre aussi le patrimoine culinaire pour lequel la traduction joue un des rôles essentiels dans le multiculturalisme aujourd'hui.

Deux contes représentent la base de mon étude: elles concernent une spécialité vietnamienne populaire: le phở. C'est une soupe préparée avec des pâtes de riz, fines et ornée d'épices variées et de viande de bœuf, cuite ou saignante; le bouillon est le point clé de la recette. Le premier raconte une histoire émouvante des deux pères qui s'occupent de leurs enfants affectueusement, et il utilise le thème de phở comme toile de fond. Le deuxième décrit la soupe phở et ses variantes.

«Phở bò nhé? Tái hay chín?» (Phong Điệp)

«Le phở au bœuf, hein? La viande juste passée dans l'eau bouillante, ou bien cuite ?» Pourquoi ne pas traduire «tái» par «saignant» au lieu de «passée dans l'eau bouillante»? Cette différence réside dans le mode de cuisson.

Comment surmonter les obstacles linguistico-culturels pour parvenir à une bonne traduction?

Mots-clé: Traduction - culture/linguistique - phở/soupe vietnamienne - convergence/ divergence

Ав STRACт: The translation of Vietnamese specialties or recipes is important since there is a very large community of Vietnamese who immigrate to France. Sharing the other's culture also includes culinary heritage in which translation plays an essential role in multiculturalism nowadays.

Two short stories represent the basis of my study: they concern a popular Vietnamese specialty: the $p h o$. This is a soup made of thin rice noodles decorated with different spices and beef, either cooked or rare; broth is the key point. The first one, which uses the theme of phở as a backdrop, tells a moving story of the two fathers who deeply care for their children. The second describes the phở soup and its various recipes. «Phở bò nhé? Tái hay Chính?» 
«Le phở au bœuf, hein? La viande juste passée dans l'eau bouillante, ou bien cuite ?» Why not translating tái by saignant (rare) instead of passée à l'eau bouillante? The adjective saignant (rare) is general whereas the past participle expression passée à l'eau bouillante is more precise: this difference lies in the cooking method.

How to overcome the linguistic and cultural barriers to achieve an accurate translation is my objective in this article.

KEY WORDs: Translation - culture/linguistics - phở/Vietnamese soup - convergence/ divergence

\section{La Soupe Phở comme toile de fond de contes et sa traduction EN FRANÇAIS}

La traduction des spécialités ou des recettes vietnamiennes est importante dans la mesure où il y a une communauté très importante de Vietnamiens qui immigrent en France. Partager la culture de l'autre recouvre aussi le patrimoine culinaire pour lequel la traduction joue un des rôles essentiels dans le multiculturalisme aujourd'hui.

Les deux contes concernent une spécialité bien connue au Vietnam: le phở. Lorsqu'on parle de «phở», on dit simplement «soupe vietnamienne». Les Français traduisent le phỏ populaire soupe vietnamienne. Le malheur est qu'au Vietnam, des soupes vietnamiennes, il y en a des tas. Si l'on traduit toutes les soupes qui y existent par soupe vietnamienne, il ne signifie rien. Pour cela, en disant soupe vietnamienne, il faut ajouter le nom propre à ce mets, le Phở pour le distinguer. Le nom des soupes varie selon le type de pâtes (pâtes de riz, pâtes de blé, etc.), coupées finement ou largement. Mieux encore, il faut le goûter.

Pour comprendre quels types d'obstacles linguistiques et culturels qu'on rencontre, je vais me baser sur deux contes bilingues vietnamiens - français traduits par Hurinville, Guy et Lo-Cicero ${ }^{1}$.

Le premier conte, de Phong Điệp ${ }^{2}$, Bát Phở / Bols de Phở concerne le client qui est en train de manger son bol de phở. Par curiosité, il regarde le bol de phở sur la table de ses voisins: les deux pères commandent deux phở pour leurs fils; ils vivent la campagne et ils partent à la capitale Hanoi pour les accompagner à leurs examens. Les pères se sacrifient toute leur vie pour leurs fils; ils ne mangent pas pour économiser leur argent. Le narrateur nous raconte cette histoire d'une manière touchante.

\footnotetext{
${ }^{1}$ Hurinville, Guy et Lo-Cicero 2015.

2 Phong Điệp 2007.
} 
Le deuxième conte de Vũ Bằng³, «Phở Bò, Món quà căn bản/ La soupe Phở, une gourmandise populaire», décrit en détails comment apprécier une vraie bonne soupe de phở, une spécialité connue dans le nord Vietnam.

Les deux contes sont choisis pour la traduction par mon groupe de travail (cf. Références bibliographiques) et il est très intéressant d'observer la complexité dans l'opération de traduction par soi-même, du vietnamien en français.

Comment surmonter les barrières linguistiques et culturelles lorsqu'il s'agit de mets ou d'ingrédients qui n'existent qu'au Vietnam?

\section{LE VietNAMiEN, SOUS INFLUENCES CHINOISE ET ROMANE}

J'aimerais faire un rappel historique concernant le vietnamien. Pendant plus de 1000 ans, le Vietnam a vécu sous l'influence de la culture chinoise. Depuis le IX ${ }^{\mathrm{e}}$ siècle avant J.C., époque à laquelle le territoire du Vietnam fut annexé par la dynastie chinoise des Triêu, jusqu'au XX $X^{e}$ siècle, le Hán (langue chinoise classique) a été utilisé dans l'administration et dans l'enseignement. Ce sont les caractères chinois qui ont constitué la première écriture vietnamienne. Ainsi, au cours des siècles, on note deux genres de poésie qui se sont développés au Vietnam: la versification suivant les règles de la prosodie chinoise, qui constitue une des épreuves au concours mandarinal, et la versification populaire opposée en tout genre au formalisme de la première. Nous avions besoin de consigner par écrit ce parler, ces idées, ces sentiments populaires, et grâce à ces besoins, nous devions nous recourir à une écriture autre que le chinois, langue étrangère et savante. De là, nous voyions naître le Nôm, la transcription des sons vietnamiens en caractères calqués sur le modèle Hán. Le Nôm se situe au XIII ${ }^{\mathrm{e}}$ siècle.

Le seul point commun entre le vietnamien et le français est l'écriture basée sur l'alphabet latin, et pour cause: le missionnaire jésuite français, Alexandre de Rhodes ${ }^{4}$, a eu un rôle historiquement fondamental et déterminant: il a donné naissance à l'écriture du Quốc Ngữ. Il a compilé, amélioré et systématisé les systèmes de transcription de ses prédécesseurs missionnaires, notamment Francisco de Pina, entre 1624 et 1644 . Son premier ouvrage imprimé latinisée actuelle est un dictionnaire annamite (ancien nom pour vietnamien) -portugais-latin paru en 1651, reprise d'un dictionnaire portugais portugais-français de Gaspar do Amaral et António Barbosa. C'était une œuvre collective internationale; toutefois, le rôle du Portugal reste peu connu encore - notamment l'apport important de Francisco de Pina, un des missionnaires portugais dans le projet de création du Quốc Ngữ. Cette écriture, qui initialement s'utilisait

\footnotetext{
${ }^{3}$ Vũ Bằng 1952.

${ }^{4}$ 1591-1660.
} 
pour l'évangilisation des premiers catholiques vietnamiens, a été utilisé par les Français et les intellectuels dans l'administration.

Avec la période française au Vietnam, le rôle de l'écriture Hán (chinoise) sera réduit, et c'est le Quốc Ngữ qui domine, à savoir un modèle de transcription en caractères latins des mots vietnamiens. Le chử Nôm ('écriture du Sud', fabriqué sur le modèle chinois) qui n'a jamais été reconnu comme écriture officielle sauf deux périodes correspondant aux deux règnes éphémères des Hồ (1400-1407) et des Nguyễn Tây Sơn (1788-1802). Pendant la colonisation française, il y avait deux écritures en cohabitation et en concurrence: le chữ Hán (l'écriture chinoise) du côté de la Cour impériale vietnamienne, et le chữ Quốc Ngữ (l'écriture de l'alphabet latin) du côté français.

Le Quốc Ngũ est devenu notre écriture nationale, celle du vietnamien. L'écriture fut présentée dans toutes les instances; Hô Chi Minh, l'ancien président du Nord Vietnam, en généralisa son emploi à partir de 1945 dans le cadre de sa campagne d'alphabétisation ${ }^{5}$.

Cette présentation est indispensable pour comprendre brièvement l'origine de l'écriture vietnamienne sous influences chinoise et romane.

Voici quelques exemples de mots empruntés à la langue française dans les deux contes: đi ôtô: aller en auto; trong tích-tắc: en une fraction de seconde, en un tic-tac; cà-rốt: carotte; mùi soa: mouchoir; foocmon: formol; mấy tấm tôn: quelques tôles.

(1) Trong tich tắc, hai bát phở sóng sánh được bê ra, đặt trịnh trọng trên bàn. (Phong Điệp)

En deux temps trois mouvements, deux bols pleins à ras bord de phở frémissant furent apportés et posés avec solennité sur la table.

L'expression «trong tích tắc/ dans un tic-tac», «tích tắc / tic-tac» dérive du son du déplacement de l'aiguille des secondes en français. Cette onomatopée est devenue un nom commun. La langue vietnamienne l'a empruntée au français et l'a assimilée ou transposée à la langue vietnamienne avec son orthographe et sa transcription phonétique: tích tắc.

On constate qu'en français, on peut traduire cette expression par «en un tic-tac» ou bien «en une fraction de seconde». L'expression «en deux temps trois mouvements» est une autre option qui signifie le même sens, c'est-àdire «rapidement».

La difficulté (ou la facilité) de la langue vietnamienne réside dans sa morphologie. C'est une langue isolante, analytique, où tous les mots restent invariables quelle que soit leur fonction syntaxique.

\footnotetext{
${ }^{5}$ Minh Ha Nguyen, 1998.
} 


\section{3. ÉTUDES LINGUISTIQUES ET CULTURELLES EN CONTEXTE DE LA LANGUE} SOURCE, LE VIETNAMIEN

\subsection{Entre dit et non-dit: de l'ellipse à l'implicite l'économie de la langue $^{6}$ vs traduction complète obligatoire}

Pour comprendre le sens de tous les mots, l'élément du vécu et du savoir culturels, voire les connaissances culinaires, est la base pour mener à bien l'opération traduisante. Les exemples qui vont suivre illustrent ce fait:

(2) Bánh cũng mềm, thơm, đảm bảo không không foocmon. (Vũ Bằng)

Vermicelle de riz - aussi - tendre - odorant - garantir - zéro - zéro - formol (Traduction Littérale: TL)

Le vermicelle de riz est tout aussi tendre, odorant et garanti sans conservateur.

À propos du terme «bánh», il faut comprendre qu'il possède plusieurs significations: 'gâteau' est le sens général. Dans ce contexte, le sens de "gâteau» est inapproprié. C'est le sens de "pâte», mais pas n'importe quelle pâte; c'est un genre de pâte de riz. «Pâte» dans ce contexte est le sens de nouille, assez large. Le mot «vermicelle ou pâte» est le plus adapté lorsqu'on parle de phở. Pour saisir le mot «bánh» utilisé ici, le contexte entier est nécessaire pour bien l'interpréter. Le titre de cette nouvelle nous l'indique: c'est «Bát phở / Les bols de phở». Il ne s'agit donc pas de «gâteaux». Pour bien interpréter le mot «bánh», il faut connaître et goûter cette soupe vietnamienne, une des soupes les plus connues et populaires dans ce pays. Cette fameuse soupe est constituée, grosso modo, de vermicelles (ou de pâtes), de riz (couleur blanche), car il existe infiniment de vermicelles ou de pâtes, et plusieurs morceaux de viande de bœuf. Le terme "phở» précise quel genre de pâte il faut utiliser. Lorsqu'on dit «bánh» dans ce contexte, cela sous-entend «pâtes» à base de farine de riz.

Plusieurs catégories de désignations ou lexicales utilisées dans les soupes vietnamiennes vont déterminer de quel type de pâtes il s'agit:

phở: soupe de vermicelles de riz, couleur blanche, grandes bandes mì: soupe de nouilles au blé, couleur jaune, grandes et petites bandes bún: pâtes de riz, se mange froid, avec divers ingrédients et de la viande de porc,

\footnotetext{
${ }^{6}$ Adam 2005.
} 
de poulet, du poisson, du crabe, etc.

miến: soupe de pâtes de riz, de manioc, de haricots, etc.

bánh canh: sorte de grosses pâtes de riz rondes, couleur blanche

bún tàu: pâtes d'un type de riz ordinaire (gạo tẻ), etc.

Toutes ces sortes de pâtes se mangent différemment selon leurs caractéristiques. Afin de bien traduire le mot "phở», il faut bien connaître la gastronomie vietnamienne. Dans plusieurs dictionnaires bilingues vietnamien - français, phở» se traduit par «soupe chinoise» qui est une grave erreur. Faute d'équivalent, on dit «soupe vietnamienne ou soupe chinoise». La meilleure solution serait de garder le nom de la soupe en tant que tel «le phở». D'ailleurs, les spécialités connues d'un pays se caractérisent par l'originalité de leurs noms propres, ici, les noms de spécialités culinaires.

On constate donc le «non-dit» qui sous-entend tout le sens du terme «bánh» grâce au contexte. «Bánh» fait partie du mot composé «bánh phở» qui signifie "pâtes de riz».

Plusieurs exemples de cette classe illustrent ce phénomène. Pour traduire ce fait de langue vietnamien en français, il faut expliciter pleinement à l'aide de paraphrases. On l'a observé dans le premier exemple: «Bánh» se traduit: «vermicelle/pâte de riz».

(3) «Bác ăn gì? Phở bò nhé. Tái hay chin? Thêm cả quả trứng cho chắc bụng nhé!» (Phong Điệp)

Vous voulez manger quoi? Alors, des soupes au bœuf, la viande juste passée dans l'eau bouillante, ou bien cuite? Et puis des œufs, pour que ça cale bien, hein?

«Vâng, gì cũng được» (Phong Điệp). Oui, ça ira très bien.

"Thế thì cho hai phở bò chín. Hai trứng.»

Alors deux soupes au bœuf, la viande bien cuite, avec deux œufs, c'est parti ! firent les pères.

Thằng nhỏ chạy bàn vo cái khăn trong tay, tân ngân nhìn bốn người, ý chừng như muốn hỏi?

Voyant les quatre personnes, le petit serveur, l'air hésitant, roulait le chiffon dans sa main; on aurait qu'il avait envie de demander:

«Sao bốn người mà chỉ có hai bát?»

Pourquoi seulement deux bols pour quatre?

Le petit serveur prend la commande rapide: «Tái hay chín? (saignant ou cuit)/ la viande juste passée dans l'eau bouillante, ou bien cuite?» Le non-dit sous-entend qu'on se réfère à de la viande de bœuf. D'ailleurs, le petit serveur résume la commande à la fin du dialogue. La traduction vers la langue cible exige l'ajout du non-dit, l'implicite de la langue source. 
(4) Nó chạy ra chỗ ông chủ quán, chỏng lỏn: (Phong Điệp)

Il courut vers le patron et annonça:

«Hai bò!»

Deux phở au boenf, deux!

«Hai bò» hors contexte peut signifier «deux bœufs». Cependant, pour différencier la viande de l'animal, la présence d'un classificateur «con» est indispensable; hai con bò: deux vaches ou bœufs. "Con" précédant un nom d'animal dénote que c'est un être animé tel que «con người, con bò, con mèo/ homme, bœuf, chat». Comme on peut le constater, le classificateur «con» ne peut être omis que dans les deux cas suivants: a. La commande au restaurant (dans le contexte ci-dessus) ou au bar; b. Les vers dans la poésie. Dans tous les autres cas, l'emploi de «con» est obligatoire.

Nous sommes donc dans un restaurant de soupe phở; le mot «phở» peut être omis. C'est l'économie de la langue. En français, en revanche, cette expression «hai bò» doit être paraphrasée: «Deux phở au bœuf, deux!»; la formule est très caractéristique d'une commande en français dans un restaurant populaire.

Revenons à l'exemple (2):

(2) Bânh cũng mềm, thơm, đảm bảo không không foocmon. (Vũ Bằng)

Vermicelle de riz - aussi - tendre - odorant - garantir - zéro - zéro - formol (Traduction Littérale: TL)

Le vermicelle de riz est tout aussi tendre, odorant et garanti sans conservateur.

En deuxième lieu, à propos de la traduction «không không foocmon». En réalité, c'est une formule: formol = 0, c'est-à-dire «pas de formol / formol nul». «Không» revêt deux sens: la négation «ne ... pas, sans» et le chiffre «zéro» (0). Le terme «formol» a été emprunté à la langue française et transposé en vietnamien: le «f» n'existe pas dans l'écriture vietnamienne, et le son «ooc» et la fin syllabique /mon/ sont la transcription phonétique du mot formol. Ce terme bisyllabique est un mot étranger français. Pour respecter la prononciation française presque telle quelle, il fallait un système logique: les syllabations fermées françaises /for-mol/, terminées respectivement par/r/ et /1/ n'existent pas en vietnamien, car presque toutes les syllabations vietnamiennes sont fermées. Il n'a pas été traduit par formol mais par conservateur ${ }^{7}$. Le formaldéhyde formol peut être présent à faible dose dans l'alimentation humaine: il apparaît naturellement dans certains aliments, tels que des fruits et des légumes, à des concentrations pouvant varier de 3 à $60 \mathrm{mg} / \mathrm{kg}$. Répertorié

\footnotetext{
${ }^{7}$ Le formaldéhyde (le formol). Dictionnaire Petit Robert 1, 1989.
} 
comme additif (conservateur) sous le code E240, il est désormais interdit en tant que tel dans l'Union européenne.

En vietnamien, formol a été emprunté au français, un terme chimique; d'ailleurs, «không, không» sont ajoutés au mot formol qui signifient «sans ou la négation «ne...pas, zéro»: pas de formol du tout et qui s'interprète comme sans conservateur.

Comme l'observe bien Ladmiral:

"Incidemment, il arrive souvent qu'une telle unité terminologique se signale de par sa facture morpho-lexicale comme un mot savant, ne füt-ce que par la grâce de son étymologie gréco-latine, plus ou moins ésotérique. Il arrive assez souvent quion ait affaire à des internationalismes.»

Il existe trois produits que les cuisiniers mettent souvent dans les aliments pour améliorer la soupe, le «hàn the» (le nom français borax), le conservateur et le glutamate de sodium (un exhausteur de goût très prisé dans la cuisine asiatique en général) ${ }^{9}$.

Pourquoi l'existence de la substance formol? En fait, cette substance garde frais les aliments tels que le bánh phở, les pâtes de riz pour cette soupe. Cependant, de nos jours, les deux produits cités sont strictement interdits, le borax et le formol qui nuisent à la santé. Pour montrer que les restaurants sont de haute qualité, il faut préciser que ces produits ne sont pas présents dans les aliments.

En français, le terme équivalent est conservateur, terme générique qui peut recouvrir plusieurs types de conservateur. Par définition, un conservateur alimentaire est une matière ajoutée aux aliments afin d'améliorer leur conservation.

Dans le deuxième conte «Phở Bò: Món Quà Căn Bản / La soupe phở $\mathrm{au}$ bœuf, une gourmandise populaire», l'étude d'une bonne soupe de phở $\mathrm{y}$ est décrite minutieusement, notamment le bouillon.

(5) Người ta bảo rằng phần nhiều những hàng phở mở hiệu như thế, nước dùng không được ngọt, hoặc có ngọt là cái ngọt của mì chính, chứ không phải là cái

\footnotetext{
${ }^{8}$ Ladmiral 2012:15
}

${ }^{9}$ https://fr.wikipedia.org/wiki/Borax. Le GMS pur n'a pas un goût agréable s'il n'est pas associé à une odeur savoureuse. En tant qu'arôme et dans la bonne proportion, le GMS peut améliorer d'autres composants actifs du goût équilibrant et arrondissant le goût global de certains plats. Il se marie bien avec la viande, le poisson, la volaille, de nombreux légumes, les sauces, les soupes et les marinades, et augmente la préférence globale de certains aliments tels que le consommé de bœuf. 
ngọt của xương bò, ấy chưa nói rằng lại còn cửa hiệu phở quá vụng về muốn có nước dùng ngọt lại cho đường vào nữa. (Vũ Bằng)

On dit que la plupart des petits restaurants populaires ou bien des cuisines de rue, propose un bouillon pas vraiment goûteux; ou bien s'il est goûteux, c'est le goût du glutamate, et non des os de bœuf, sans compter les maladroits qui parfument le bouillon en ajoutant du sucre.

(6) Ông chủ quán - đứng giữa hai cái bếp than rừng rực cháy, hai cái nồi nước sùng sục sôi và chỉ có mỗi cái khăn cháo lòng vắt vai để vuốt đi những lượt mồ hôi nhễ nhai. Hẳn sẽ nổi sung lên. (Vũ Bằng)

Le patron, debout entre deux feux de cuisine remplis de charbons ardents et deux marmites d'eau bouillante, un torchon grisâtre usé sur l'épaule lui servant à éponger la sueur qui l'inonde, pourrait se fâcher.

Dans l'exemple (6), le syntagme nominal «cái khăn cháo lòng / classificateur objet + torchon + soupe de riz + tripes» est laborieux pour les Occidentaux dans son interprétation: un nom commun juxtaposé à une spécialité culinaire vietnamienne: soupe de riz aux tripes. On conçoit difficilement ce rapport sémantique entre les deux termes. Seuls les natifs de la langue saisissent le rapprochement entre les deux mots.

Le rapport entre le torchon et la soupe de riz aux tripes est la couleur du torchon, la couleur jaunâtre de la soupe de riz aux tripes. Le torchon est sale et sa couleur est jaune à cause de son usage fréquent. La traduction ne peut être rendue en tant que telle car le lecteur occidental n'a pas le même référent culturel que le natif vietnamien. À cette fin, le traducteur doit substituer le même sens de l'expression originale à celui de la langue cible, le français. Dans l'exemple (6), la traduction n'a pas pu être faite littéralement à cause du référent culturel différent du vietnamien en français. Seule la traduction sémantique reste possible: le torchon grisâtre (le torchon à la couleur de la soupe de riz aux tripes).

L'exemple (7) présente une seconde fois cette expression, et elle est plus explicite: cái khăn màu cháo lòng / classificateur objet - torchon - couleur - soupe de riz - tripes (le torchon couleur de la soupe de riz aux tripes). La comparaison est intéressante car elle met en rapport le torchon avec une spécialité de soupe vietnamienne, couleur jaunâtre.

(7) Ông chủ quán lăn cái khăn mầu cháo lòng qua vuông mặt đỏ lựng vì lửa than, rồi vắt trả cái khăn về bên vai, tay dao tay thớt nhoay nhoáy. (Phong Điệp)

Le patron du restaurant jeta le torchon crasseux en travers de son visage tout rouge à cause du feu, puis le replaça sur l'épaule; couteau et hachoir volèrent à la vitesse de l'éclair.

À l'usage, le torchon est devenu jaunâtre. Le savoir et le vécu sont nécessaires pour bien expliquer cette expression. La traduction littérale, dans 
ce contexte, ne va pas être possible car le lecteur français ou occidental ne pourra pas saisir le sens de cette expression; la soupe de riz aux tripes n'est pas un plat français.

(8) Sẽ là một đĩa cơm suất ven đường, ít thịt, nhiều rau, xin thêm miếng cháy cho chắc dạ. (Phong Điệp)

Pour eux ce serait une portion de riz dans une assiette au bord de la route, peu de viande mais plus de légumes, on demanderait en outre une portion de riz en crôte pour garnir l'estomac.

Một đĩa cơm suất / une portion de riz dans une assiette: cette expression n'est pas, en réalité, quelque chose d'inhabituel. Néanmoins, quelques observations doivent être faites. En général, les Vietnamiens mangent du riz dans un bol, avec des baguettes, chose commune. Cependant, il existe souvent des restaurants qui servent du riz dans une assiette avec différents aliments et des couverts occidentaux: cuillère et fourchette. D'une manière générale, ce genre de service existe dans des restaurants populaires, pour simplifier l'addition; pour cela, on dit một đĩa co'm suất: une part d'assiette de riz.

Dans le même exemple, il y a l'expression miếng cháy / une portion de riz en croûte. Pour les Occidentaux, il est inhabituel de manger du riz brûlé. Au Vietnam, généralement, on cuisine le riz dans une poêle; lorsque l'eau bout, il faut réduire la flamme à feu très doux pour continuer la cuisson jusqu'à obtenir au fond de la poêle, une couche de riz un peu brûlé en croûte, que l'on mange avec de la sauce de poisson (nước mắm, sauce de base dans la cuisine vietnamienne), de la cive à l'huile ou à la graisse, recette méconnue des Occidentaux.

(9) Hành chần tới độ, không hăng mà vẫn giòn, ngọt. (Vũ Bằng)/ L'oignon a été poché dans les règles de l'art, il n'est plus âpre, mais croquant et doux.

Dans l'exemple (9), sa traduction littérale est: oignon + plonger dans l'eau bouillante rapidement + jusque + degré adéquat. Le syntagme verbal chần tới độ ne comporte que trois mots. Mais ces mots doivent être très bien interprétés pour être traduits: la forme verbale englobe tout le sens de "plonger dans l'eau bouillante»; quant au groupe de mots tó i độ couvre toute l'idée: jusqu'à un degré approprié, pour que l'oignon ne soit pas trop cuit et qu'il reste croquant et doux. La langue vietnamienne est très économique. Tới độ signifie en fait jusqu'au bon degré, jusqu'au degré adéquat, c'est-à-dire à la bonne température. Le lecteur vietnamien devine aisément le sous-entendu, le non-dit de l'expression littérale Tới độ / jusqu'au degré. En français, on peut la traduire par: lorsque l'oignon est plongé dans l'eau bouillante jusqu'à un degré adéquat (...). L'oignon a été poché dans les règles 
de l'art résume tout à fait cette idée en vietnamien. On remarque la forme verbale être poché qui signifie être plongé rapidement dans l'eau bouillante. La forme verbale vietnamienne chần tới độ est l'équivalent du français être poché dans les règles de l'art.

(10) Người cha sẽ đợi những đứa con của mình ăn đến tận thìa nước cuối cùng, rồi lẳng lặng moi cái ví bằng vải bông chần màu lam, cất trong ngực áo ra. (Vũ Bằng) Les pères attendaient patiemment que leurs fils eurent fini leur dernière cuillerée de soupe, et discrètement, ils sortirent des portefeuilles en piqué de coton bleu, dissimulés sous leur chemise.

Dans cette expression thìa nước cuối cùng (cuillère + eau + dernier), le mot soupe est omis. Sans le contexte, on peut l'interpréter par dernière cuillerée d'eau ou dernière cuillère d'eau» car nuó́c signifie aussi eau. Mais, dans ce cas précis, il s'agit naturellement de la dernière cuillerée de la soupe phỏ.

Pour bien interpréter le sens complet de chaque expression du vietnamien en français, il faut être bilingue, et savoir paraphraser lorsqu'il est nécessaire. Nous l'avons vu, plusieurs non-dits figurent dans le discours, ou plusieurs expressions vietnamiennes ne peuvent se traduire en tant que telles, alors, il faut toujours recourir aux procédés linguistiques variés afin de bien interpréter les caractéristiques linguistiques et culturelles vietnamiennes qui n'existent aucunement ou qui n'ont pas d'équivalents en français.

Comme le souligne très bien Adam ${ }^{10}$ :

"L'incomplétude est la règle du discours, en vertu d'une loi d'économie du langage qui permet de ne pas tout dire (opération d'abrègement) et d'impliciter ce que l'auteur ou le lecteur peuvent restituer facilement etlou inférer sur la base de diverses formes d'implicite."

Le dernier exemple, intéressant à observer du point de vue sémantique, à propos du titre du deuxième conte: Phò bò, món quà căn bản/La soupe de phỏ, une gourmandise populaire. Le mot quà revêt plusieurs sens: cadeau, friandise. Il m'est très difficile de le traduire car les deux sens peuvent être bien adaptés dans le présent contexte. Cadeau est hors de question. Il serait inapproprié de le traduire par «friandise»: il concerne plus les desserts sucrés, tels que les gâteaux, les biscuits. Ce n'est ni une friandise car, d'après sa définition, une friandise est une sucrerie, une petite pièce de confiserie ou de pâtisserie. La proposition pourrait être le mot plat; il s'adapte bien. Toutefois, la lecture de ce conte m'aide à mieux interpréter le mot món quà. On parle de différents plats de desserts sucrés. Mais, pour bien signifier que le phở, un très bon plat, on

\footnotetext{
${ }^{10}$ Adam 2005: 111.
} 
le mange avec plaisir comme si c'était une gourmandise. En ce qui concerne le mot căn bản qui signifie (de) base: il implique un plat populaire. On peut le manger à n'importe quel repas: petit-déjeuner, déjeuner, goûter ou dîner.

Dans l'opération de traduction toutefois, il serait impossible de transposer ces non-dits, ces phénomènes implicites d'une langue à l'autre, causés par les spécificités linguistiques et culturelles. Le lecteur français ne pourra pas saisir le vrai sens étant donné que ses présupposés ne correspondent aucunement à ceux d'un lecteur vietnamien, et en plus traduit tels quels dans la langue cible. Ses repères seront entièrement perdus. Aussi, le traducteur, qui maîtrise parfaitement sa langue et sa culture, essaiera-t-il de donner le plus d'informations et de repères linguistiques et culturelles possibles pour transposer les idées de la langue source à la langue cible, sans pour autant dénaturer ses spécificités linguistico-culturelles. Ladmiral parle d'ailleurs de «Conflit des Interprétations ou bien d'interprétations conflictuelles.» ${ }^{11}$

\subsection{Quelques points sur la morphosyntaxe du vietnamien en français}

La morphosyntaxe du vietnamien est aux antipodes de celle du français ou du portugais. Pour savoir la classe d'un mot, il faut l'analyser dans le contexte même. Un nom, un verbe, un adjectif se déterminent grâce à leur position dans la phrase. Le mot phỏ, juxtaposé ou précédé d'un terme, va donner la signification précise, dépendant du repère du nom noyau dans le syntagme nominal dans la phrase.

Hàng phở: petit restaurant de phở - parler du sud

Quán phở: petit restaurant de phở - parler du nord

Bát phở: bol de phở - parler du nord

Tô phở: bol de phở - parler du sud

Phở bò: phở au bœuf

Phở tái: phở au bœuf saignant

Phở chín: phở au bœuf cuit

Le deuxième terme (souvent un épithète) modifie ou qualifie le premier terme. En fonction du contexte et/ou de la position d'un terme, on va saisir le sens correct. Néanmoins, parfois, on a affaire davantage au style comme c'est le cas de «Phở hiệu» (mot sino-vietnamien) ou bien «Hiệu phở» (mot purement vietnamien) qui signifie «restaurant de Phở».

Observons également, dépendant toujours du contexte, «Phở hiệu»a un autre sens: il s'agit du phở commandé au restaurant qui s'oppose au phở nấu ở nhà (phở fait maison).

\footnotetext{
${ }^{11}$ Ladmiral 2012: 45.
} 
Il est toujours ardu de définir d'emblée si le mot est un adjectif, un verbe, une préposition, etc. Pour ce faire, il faut le situer, l'analyser dans un contexte particulier. Pour cette raison, le vietnamien se définit comme une langue isolante, analytique.

\subsubsection{Comment interpréter le mot "phở» dans son contexte}

Le phở est une soupe aux nouilles de riz appelée bánh phở d'où son nom phở.

(11) Trong tích tắc, hai bát phở sóng sánh được bê ra, đặt trịnh trọng trên bàn. (Phong Điệp)

En deux temps trois mouvements, deux bols pleins à ras bord de phở frémissant furent apportés et posés avec solennité sur la table.

Hai bát phở sóng sánh / deux bols pleins à ras bord de phở frémissant joue le rôle d'agent qui subit l'action (les bols de soupe furent apportés). L'explication syntaxique se réalise selon la syntaxe française. Il me semble plus aisé de l'expliquer ainsi. Hai bát phở / deux bols (de) phở: c'est un nom composé dans lequel «bát» est le nom noyau et «phở», son épithète.

(12) Bạn có bao giờ, khi đang ngồi trong một hàng phở, và đặt ra câu hỏi: có gì lạ với bát phở của người kế bên mình? (Phong Điệp)

Dans un restaurant de phở, vous êtes-vous jamais posé la question: «Est-ce que mon bol de phở est différent de celui de mon voisin?»

Dans l'exemple (12), il y a deux occurrences de phở: hàng (nom noyau) phở (restaurant de phở) et bát (nom noyau) phở (bol de phở). Lorsqu'on juxtapose phở, nom noyau, à un autre nom commun, il joue le rôle de l'épithète.

(13) Phở bò (soupe-bœuf): Soupe de phở au bœuf (phở: nom noyau)

Người ăn phở (personne - manger - phở): client de phở (Người ăn: mot composé, nom noyau)

Người sành phở (personne - connaître - phở): connaisseur de phở (Người sành: mot composé, nom noyau)

(14) Anh phở Tráng (monsieur ou frère - phở - Tráng (nom propre): le restaurateur Tráng - chez Tráng (Anh phở: mot composé, nom noyau)

Ce sont des syntagmes nominaux dans lesquels il existe toujours le nom noyau. Pour comprendre la syntaxe de chaque groupe nominal, la traduction littérale est nécessaire (voir ci-dessus). L'essentiel est de distinguer le nom noyau dans la phrase et de l'interpréter. 
Pour l'exemple (13), anh désigne une personne au masculin. Il est dérivé d'un terme de lien familial: frère aîné. Il est devenu un terme de relation sociale. Un restaurateur du restaurant Phở Tráng se dit anh phở Tráng / Monsieur Trang du restaurant phở. On peut simplement le traduire par Chez Phở Tráng.

Les formes verbales sont plus aisées à interpréter grâce à leur sémantisme:

(15) Ăn phở: manger la soupe phở

Xơi phở: apprécier la soupe phở

Nấu phở: préparer le phở

Bán phở: vendre de la soupe phở

Mua phở: acheter de la soupe phở

Au Vietnam, il existe plusieurs catégories de restaurants, selon de leur taille, leur importance ou leur mode de vendre. Les mots entre guillemets sont les noms noyaux:

«Quán» phở: restaurant populaire de phở

«Tiệm» phở: petit restaurant de phở

«Hàng phở» xe: chariot ambulant de soupes phở

(16) «Phở» hiệu: restaurant de phở / hiệu phở

«Phở» gánh: phở ambulant

«Gánh» phở: porter le phở / le phở ambulant

En fonction du contexte et/ou de la position du terme noyau, on va saisir le sens correct. Dans le cas de phở hiệu ou hiệu phở, que phở précède un autre mot ou soit juxtaposé, le sens ne change pas; ici, on a affaire davantage au style. En revanche, dans le cas de gánh phở, on l'interprète comme une forme verbale: porter/vendre la soupe de phở alors que phở gánh est plutôt un syntagme nominal dans lequel «phở» est le nom noyau (le phở ambulant): vente ambulante de phở. Le deuxième terme détermine ou qualifie le premier terme.

(17) Thì ta lại cũng thấy biết bao nhiêu người sang trọng lần mò tới chỗ rất tồi tàn để ăn cho được một hai bát phở.

(TL: alors - nous - encore - aussi - voir - plusieurs - personnes - luxueux chercher - endroit - très - pauvre - sale - pour - manger - pouvoir - un - deux - bols - soupes - phở)

Alors, on peut voir aussi combien de gens riches cherchent et trouvent les endroits modestes pour pouvoir manger un ou deux bols phở.

Đó là do người ăn phở sành, hầu hểt, chỉ chủ tâm đến cái điểm chính là phở mà thôi, chứ không quan tâm đến ngoại cảnh làm gi. (Vũ Bằng)

(TL: ça - est - parce que - personne - manger - phở - connaisseur - la plupart - seulement - concentrer - sur - cl. objet - point - vis-à-vis - principal - est 
- phở - particule de «ne... que» - mais - ne pas - concerner - préposition extérieur - paysage - faire - quoi)

Parce que c'est un vrai connaisseur de phở; la plupart des connaisseurs ne se concentrent que sur la soupe phở, et n'accordent aucune attention à l'aspect extérieur du restaurant.

Une langue isolante telle que le vietnamien se décrit de la manière suivante: un même signe, une même graphie peut remplir différentes fonctions, selon la place qu'il occupe dans une phrase, dans un discours donné. Il a une double valeur: sémantique et grammaticale ${ }^{12}$. La traduction littérale de chaque terme aide le lecteur à comprendre la phrase. Un mot a par conséquent un sens, une nature et une fonction syntaxique.

Groupe: verbe + nom

Ăn phở: manger de la soupe phở.

Gánh phở: apporter, porter la soupe phở

Đi mua phở: aller acheter de la soupe phở

Nom + nom

Tô phở: bol de soupe phở

Phrase: Pronom + verbe + nom complément

Tôi ăn phở. Je mange de la soupe phở

Nom + nom

Mùi phở: l’odeur/le parfum du phở

Le rapport logique entre les mots détermine leurs fonctions grammaticales. On peut résumer comme suit:

- d'un groupe nominal sujet (article, substantif, adjectif),

- d'un groupe verbal (des verbes d'action, d'état, de devenir, etc.)

- d'un groupe nominal complément (compléments circonstanciels, de nom, d'objet, d'adjectifs, d'adverbes).

Lordre de la phrase est:

Groupe nominal sujet + Groupe verbal + (Groupe nominal complément).

(18) Lúc tôi bắt đầu ăn, họ ngồi trong quán nước trà kế bên. (Phong Điệp) (lorsque - je - commencer - manger - ils - asseoir - dans - salon - thé - voisin) Lorsque j'avais commencé à manger, ils étaient encore dans un salon de thé voisin.

(Groupe Nominal Complément Circonstanciel - Groupe Verbal - Groupe Nominal Circonstanciel)

${ }^{12}$ Nguyễn Tôn Nữ Hoàng Mai, 1998. 


\section{3. Éléments culturels de comparaison dans la langue source: composants/référents étrangers aux natifs de la langue cible}

En dernier lieu, j’aimerais présenter les éléments linguistico-culturels incompatibles du vietnamien en français. Il y a des éléments linguistiques qui ne correspondent pas ou qui n'existent aucunement du point de vue culturel d'une langue à une autre. Comment les traduire, les transposer ou bien les interpréter? Les exemples étudiés ci-dessus ont déjà témoigné cet aspect (màu cháo lòng / couleur sale; chần độ / pocher dans les règles de l'art; miếng cháy, un pain de riz brûlé, etc.)

Combler les divers obstacles linguistico-culturels par différents moyens linguistiques est une tâche complexe:

(19) Điều cần thiết là bánh phải mỏng và dẻo, thịt mềm, và nhất là nước dùng phải ngọt, ngọt kiểu chân thật, nghĩa là ngọt vì nhiều xương, tẩy vừa vặn không nồng, mà lại tra vừa mắm muối, không mặn quá mà không nhạt quá. (Vũ Bằng) Le plus important est que les vermicelles soient fins et fermes, que la viande soit tendre, et surtout que le bouillon soit parfumé naturellement, c'est-à-dire parfumé grâce aux os, suffisamment essangés; il faut aussi bien assaisonner le bouillon de phở avec de la sauce de saumure, ni trop salé, ni trop fade (MHL $\left.{ }^{13}\right)$.

Du point de vue lexico-sémantique, le terme ngọt est très difficile à rendre en français. Son sens propre signifie sucré ou doux lorsqu'on parle de desserts, leur goût sucré. Dans l'exemple 19, il serait étrange de le traduire par sucré car on parle de bouillon ou de soupe. Le goût sucré peut provenir d'autres ingrédients comme de l'oignon, des os à moelle, etc. La saveur du bouillon de phở est la base dans sa préparation. Il ne suffit pas d'y ajouter des cubes de bouillon de bœuf ou du glutamate de sodium. Il faut des heures pour le mijoter. Le goût du bouillon est spécifique: le terme parfumé est très bien adapté car ngọt exprime toute cette saveur particulière difficile à décrire.

Dans le deuxième conte, l'histoire est simple: comment apprécier une vraie soupe de phở? L'auteur y démontre plusieurs possibilités dans la réussite de la préparation du phở. Il existe des cuisiniers qui dénaturent le phở. Les comparaisons pertinentes ont été décrites pour témoigner de nombreuses innovations fantaisistes chez les cuisiniers. Une seule personne est capable de juger de la qualité d'un phở: un fin connaisseur.

Dans la société asiatique, les référents de comparaison ne sont pas les mêmes que dans la société occidentale. Comment le traducteur va remédier à ce type d'obstacle?

\footnotetext{
${ }^{13}$ MHL: Minh Ha Lo-Cicero. Je propose la présente traduction.
} 
Les différentes comparaisons sont innommables lorsqu'on parle d'un très bon phở:

(20) Thật thế, phở đối với một hạng người, không còn là món ăn nữa, mà la thứ nghiện như nghiện thuốc lào, thuốc lá, trà tươi, thuốc phiện. (Vũ Bằng)

En effet, le phở, pour une classe de personnes, n'est plus un mets, mais une sorte de toxicomanie telle que le tabac pour pipe à eau, la cigarette, le thé aux feuilles fraîches ou l'opium.

Je propose la présente traduction de l'exemple (20). Pour exprimer comment les connaisseurs sont accrocs au phở, la comparaison est intéressante: c'est une toxicomanie de phở similaire à celle de l'opium, au thé, aux feuilles fraîches ou à la cigarette. On imagine très bien le goût de l'excellent phở.

(21) Ngay từ ở đằng xa, mùi phở cũng đã có một sức huyền bí quyến rũ ta như mây khói chùa Hương đẩy bước chân ta, thúc bách ta phải trèo lên đỉnh núi để vào chùa trong rồi lại ra chùa ngoài. (Vũ Bằng)

Même de loin, le parfum de la soupe a déjà un charme mystérieux comme le nuage de fumée de la Pagode des Parfums, qui nous pousse à aller de l'avant. Il faut gravir la montagne pour pouvoir arriver à la première Pagode et ensuite aller à la deuxième Pagode.

L'exemple 21 fait la comparaison du parfum du phở avec les nuages de fumée de la fameuse Pagode des Parfums, un des plus beaux sites touristiques dans le nord du Vietnam. Une telle mise en parallèle exprime déjà la qualité gustative de la soupe, car si on connaît la Pagode des Parfums, on peut imaginer aisément l'excellente saveur de phở. Pour un lecteur occidental, la comparaison du phở avec la Pagode des Parfums ne lui dira rien. Le traducteur pourrait proposer une autre comparaison que celle de la Pagode des Parfums: l'immense Château de Versailles par exemple. Cependant, ce serait incompatible. Un lecteur occidental, pourrait quand même imaginer ce qu'est le parfum de la soupe même s'il ne connaît pas le référent culturel de la comparaison. La nouvelle culture peut être découverte à travers la langue. Aussi, les nuances de la traduction servent-elles précisément à l'approche d'une autre culture.

(22) - Ta tiến lại gần một cửa hàng bán phở, thật là cả một bài trí nên thơ. Qua lần cửa kính ta đã thấy gì? Một bó hành hoa xanh như lá mạ, dăm quả ớt đỏ buộc vào một cái dây, vài miếng thịt bò tươi và mềm, chín có, tái có, sụn có, mõ̃ gầu có, vè cũng có. (Vũ Bằng)

- Approchons-nous d'un petit restaurant de phở: un vrai décor poétique. Que voit-on à travers la vitrine? Une botte de cive d'un vert intense telles les feuilles de plants de riz, quelques piments rouges attachés à une ficelle, des morceaux de 
bœuf frais et tendres, cuits ou bien passés dans l'eau bouillante, cartilages, jarrets, rien ne manque.

La description minutieuse d'un bon petit restaurant de phở encourage les gens à y entrer: le décor est essentiel. Les différents ingrédients pour la soupe sont exposés dans la vitrine et naturellement ils attirent l'attention des passants et les invitent tacitement à entrer. La botte de cive très verte comparée à la couleur des feuilles de plants de riz, les quelques piments rouges qui contrastent avec le vert de la cive, le rouge vif de la viande fraîche et les différentes cuissons de bœuf présentés dans la vitrine sont les ingrédients clés pour le phở: c'est une invitation aux gourmets.

En revanche, un lecteur français va réagir autrement en raison de nouveaux éléments culturels. Tout le vocabulaire va devenir évocateur de la culture de l'autre. Grâce à ce paramètre important, le traducteur natif ne peut que respecter le contenu linguistico-culturel pour le transposer dans la langue cible.

(23) - Người bán hàng đứng thái bánh, thái thịt luôn tay, thỉnh thoảng lại mở nắp một cái thùng sắt ra để lấy nước dùng chan vào bát. Một làn khói tỏa ra khắp gian hàng, bao phủ những người ngồi ăn ở chung quanh trong một làn sương mỏng, mơ hồ như một bức tranh Tàu vẽ những ông tiên ngồi đánh cờ ỏ trong rừng mùa thu. (Vũ Bằng)

- Debout, le restaurateur coupe les vermicelles, et la viande continuellement, et de temps en temps, il ouvre la grande marmite en métal pour prendre le bouillon et le verser dans le bol. Un nuage de fumée s'envole, et remplit tout le restaurant d'un léger brouillard, autour les clients qui mangent, telle une peinture chinoise où l'on peint les vieillards immortels assis, en train de jouer aux échecs dans la forêt, en automne.

L'atmosphère agréable et appétissante du petit restaurant de phở est comparée à une peinture chinoise onirique. Le lecteur vietnamien connaît très bien le fameux tableau que décrit l'auteur: c'est le symbole de la sérénité, de la beauté du paysage, très connu en Asie. Cette référence à la beauté picturale est rapportée à l'atmosphère appétissante du restaurant traditionnel vietnamien: une découverte pour le lecteur français.

(24) - Có người kể chuyện rằng trước đây mười lăm, hai mươi năm, đã có một hàng phở ở phố Mới tìm lối cải cách phở, cũng như Năm Châu, Phùng Há dạo nào cải cách cải lương Nam kỳ, tung ra sân khấu những bản "De đơ dà múa" Họ cho mà dầu và đậu phụ vào phở, nhưng cố nhiên là thất bại. (Vũ Bằng)

- Des gens racontaient quinze ou vingt ans auparavant, qu'il existait un restaurant du nouveau quartier qui voulait réformer ou améliorer le phở, comme le faisaient à l'époque, les anciens artistes du théâtre rénové de renom, Năm Châu, Phùng Há, de la région du Sud du Vietnam (Nam kỳ); ils sélançaient sur la 
scène et chantaient «De đơ dà múa»! (J'ai deux amours). Eux mettaient de la sauce maggi, et du tofu dans la soupe; naturellement, cétait voué à l'échec.

La critique à propos de certains cuisiniers sur la préparation du phở concerne cette aberration culinaire. Les cuisiniers, les restaurateurs aiment bien innover en recréant la préparation du phở. Mais il y a des limites: il ne faut pas dénaturer le goût caractéristique du plat. Dans l'exemple (24), Vũ Bằng étudie le personnage du restaurateur-cuisinier qui voulait créer une nouvelle recette du phở. Il décrit l'innovation étrange du cuisinier. La comparaison saute aux yeux du lecteur natif ou non natif; pour illustrer cette aberration de créativité chez le cuisinier, l'auteur la compare à un groupe de théâtre rénové, similaire à l'opéra chinois, avec deux artistes de renom (l'acteur Năm Châu et l'actrice Phùng Há). Au lieu de présenter leur chant classique du théâtre rénové, ils chantaient "De đơ dà múa".

Cet étrange titre de chanson est en réalité la transcription phonétique vietnamienne du français «'ai deux amours» chanté par Joséphine Baker ${ }^{14}$. La mise en parallèle veut tout dire: le théâtre rénové vietnamien inclut un titre de chanson française de variétés. Les éléments de comparaison sont aux antipodes l'un de l'autre, incompatibles.

Dans la préparation de la soupe de phở, l'ajout d'une sauce (mà dầu) et du tofu est contradictoire avec les caractéristiques de la recette. La comparaison très critique de l'auteur a une forte pointe d'humour: on comprend le caractère d'incompatibilité de cette création ridicule. L'élément de la mise en parallèle avec le titre de la chanson française se justifie: c'était la période de la présence française au Vietnam, dans les années 50.

Le symbole culturel et le symbole langagier que représentent le cải lương/le théâtre rénové et «J'ai deux amours» justifient cette impossibilité. On observe bien que langue et culture sont deux systèmes sémiologiques qui s'imbriquent, indissociables. Le cải lương/le théâtre rénové appartient à la langue/culture orientale opposé à «J'ai deux amours», chanson française, langue/culture occidentale.

Un autre élément linguistique à souligner est la vietnamisation du titre de la chanson «De đơ dà múa». Pour ne pas dépayser le lecteur natif du point de vue linguistique, Vũ Bằng $\mathrm{a}$ transcrit phonétiquement le titre. Tout en donnant le titre d'une chanson française, l'auteur reste dans l'univers vietnamien. On devine naturellement que le natif sait à quoi il se réfère.

À propos de la terminologie «mà dầu»: ce mot ne figure pas dans le dictionnaire. Il est constitué de deux termes de sauce: sauce maggi et sauce

14 “J'ai deux amours", paroles de Géo Koger et Henri Varna, musique de Vincent Scotto, chantée par Joséphine Baker, 1930. 
soja. C'est la création d'un mot vietnamien à partir d'un mot français: mà provient de la sauce maggi, soit la première syllabe et dầu, qui signifie liquide ou sauce, comme dans le modèle de désignation xì dầu (sauce de couleur noire, sauce soja), la deuxième syllabe (sauce de soja) = mà dầu ${ }^{15}$. Ce terme est désuet. En revanche, le terme existe sous deux orthographes (nord et sud) «ma-gi ou ma-di [mazi]» plus actuelles.

La comparaison culturelle et langagière est logique: il est impensable d'ajouter des ingrédients autres que ceux de la préparation originelle du phở, et surtout ceux qui sont incompatibles avec la recette classique, comme la sauce mà dầu - sauce maggi - et le tofu. Toutefois, on peut comprendre l'idée de rénovation gustative chez les restaurateurs: le mélange des goûts Est et Ouest.

\section{Richesses linguistico-CUlturelles bilingues Vietnamien FRANÇAIS DANS L'UNIVERS CULINAIRE}

C'est une immense source d'informations que d'étudier ces obstacles linguistiques et culturels dans l'art culinaire, surtout lorsqu'il s'agit de deux cultures qui s'opposent: l'Orient et l'Occident que représentent le Vietnam et la France.

«Le vocabulaire, accès à la culture? À sén tenir au plan lexical ordinairement sollicité, cette réinterprétation prudente des relations vocabulairelculture consisterait, en premier lieu, à considérer que les mots ne traduisent pas directement, par leur sens enregistré en dictionnaire, les phénomènes culturels et qu'ainsi la connaissance de leurs significations tient lieu de connaissance culturelle.» ${ }^{16}$

Pour le traducteur, comme le remarque Medhat-Lecocq ${ }^{17}$ :

"Il est inconcevable aujourd'hui d'aborder ou d'appréhender une langue dans sa seule dimension linguistique. À chaque fois que l'on cherche à interpréter les signes linguistiques d'un texte, quelle que soit la langue dans laquelle celui-ci est rédigé, c'est l'extralinguistique qui nous guette et nous poursuit pour nous orienter vers la bonne interprétation du texte.»

L'opération de traduction représente un défi constant pour le traducteur. Ce dernier est, en réalité, une personne triplement spécialisée: a. le spécialiste

${ }^{15}$ D'après les précieuses éclaircissements de M. Quốc Hùng Nguyễn: ex-professeur d'anglais à l'École Supérieure des Langues Étrangères à Hà Nội (Nord Việt Nam).

${ }^{16}$ Beacco, 2000:138.

${ }^{17}$ Medhat-Lecocq, INALCO, page 85. 
émetteur et récepteur du savoir; b. le terminologue, partagé entre les deux approches normatives et descriptive et enfin c. le traducteur ${ }^{18}$.

Il y a des éléments linguistiques et culturels de la langue de départ qui sont intraduisibles car il n'existe pas d'équivalents dans la langue cible: on a affaire à des cas d'«intraductibilité». Soulignons que «langue et culture» impliquent ce que Medhat-Lecocq désigne par l'extralinguistique. Néanmoins, selon le contexte d'origine et les éléments culturels inhérents à la langue, le traducteur doit respecter l'idée de l'auteur même si les référents, les symboles linguistiques et culturels sont inconnus du lecteur français ou occidental car la langue et la culture, paramètres clés, signifient: accéder à la culture, à la langue de l'autre.

Les questions culturelles et linguistiques présentées dans cet article illustrent la complexité de l'opération traduisante telles que les entre dit et les non-dit: de l'ellipse à l'implicite, l'économie de la langue vs traduction complète obligatoire, la terminologie, la morphologie et les éléments culturels de comparaison dans la langue source: composants/référents étrangers aux natifs de la langue cible.

Il existe toujours des difficultés d'ordre culturel et linguistique dans l'entreprise de traduction. Il n'en est pas moins vrai qu'on puisse trouver de multiples solutions, différents procédés linguistiques pour atteindre l'objectif en question: faire comprendre et faire connaître la culture d'un monde différent du sien grâce aux moyens de la langue à travers les mots, les équivalents, les paraphrases, ou à travers les repères culturels de son propre pays, ou à travers les comparaisons interculturelles variées.

Lopération de traduction reflète toute la complexité linguistico-culturelle très instructive, à travers une simple spécialité populaire de soupe qu'est le phở, à la saveur singulière, difficile à décrire, ou à traduire, faute d'ingrédients correspondants d'où l'absence de terminologie. La gastronomie fait partie du patrimoine culturel de chaque pays, et elle se partage dans le monde entier grâce aux traductions, de l'Orient à l'Occident et vice versa. On aime bien goûter de nouvelles spécialités de chaque pays. La recette d'un plat spécial est le moyen idéal pour atteindre cet objectif. Les ingrédients, les produits d'un plat déterminé, d'une recette, symbolisent un pays, et font partie intégrante de la langue et de la culture, indissociables.

Mon expérience personnelle de traductrice à propos de ces deux contes sur le phở m’a permis de découvrir non seulement les spécificités culturelles et linguistiques de ma propre langue, mais aussi de percevoir et d'apprendre la complexité de la langue française à travers la gastronomie. Ces obstacles

\footnotetext{
${ }^{18}$ Ibid. page 86.
} 
résident dans les deux langues/cultures, totalement opposées l'une de l'autre: la langue vietnamienne isolante et la langue française flexionnelle.

\section{RÉFÉRENCES BIBLIOGRAPHIQUES}

Adam, J.-M. (2005), La linguistique textuelle. Introduction à l'analyse textuelle des discours. Armand Colin. Cursus. Paris.

Beacco, J.-C. (2000), Les dimensions culturelles des enseignements de langue. Hachette. F 75905. Paris.

Briu, J.-J. (éd.) (2012), Terminologie (II): comparaisons, transferts, (in)traductions. Travaux Interdisciplinaires et Plurilingues. 17. Peter Lang. Bern - Berlin - Bruxelles Frankfurt am Main - New York - Oxford - Wien.

Claver Zouogbo,J.-P. (2009), Le proverbe entre langues et cultures. Une étude de linguistique confrontative allemand / français / bété. Préfaces de Gertrud Gréciano et Annelies Häcki-Buhofer. Peter Lang. Bern - Berlin - Bruxelles - Frankfurt am Main New York - Oxford - Wien.

Dauphin, A. (1992), Cours de vietnamien. L'Asiathèque. Paris.

Ladmiral, J.-R. (2012), "La terminologie au risque de la traduction", in Briu, J.J. (éd.), Terminologie (II): comparaisons, transferts, (in)traductions. Travaux Interdisciplinaires et Plurilingues. 17. Peter Lang. Bern - Berlin - Bruxelles Frankfurt am Main - New York - Oxford - Wien, page 85 - page 102.

Medhat-Lecocq, H. (2012), "Dans l'approche culturelle: ce que le terminologue doit au traducteur", in Briu, J.-J. (éd.), Terminologie (II): comparaisons, transferts, (in) traductions. Travaux Interdisciplinaires et Plurilingues. 17. Peter Lang. Bern Berlin - Bruxelles - Frankfurt am Main - New York - Oxford - Wien, page 11 - page 20.

Nguyễn, Kim Thản, (1997), Nghiên Cứu Ngũu Pháp Tiếng Việt. Nhà Xuất Bản Giáo Dục. Hà Nội, Viẹt Nam.

Nguyễn, Tài Chình, (1981) Ngũ Pháp Tiếng Việt, Tiếng - Tù Ghép, Đoạn Ngũ (La Grammaire de la langue vietnamienne, la langue, les mots composés, la phrase), Nhà Xuất Bản Đại Học và Trung Học Chuyên Nghiệp, Hà Nội.

Nguyễn Tuân, (1957), 'Phở, báo Văn số 1 và số 2 , ngày 10-5 và ngày 17-5, Hà Nội Vũ Bằng (1957), 'Phở bò, món quà căn bản', trong Miếng ngon Hà Nội NX̉ ?, Hà Nội (Note: L'écrivain Thạch Lam et le poète Tú Mỡ étaient les premiers à introduire la gastronomie vietnamienne (dont le Phở) en prose et en poésie dans la littérature vietnamienne).

Version rééditée de ces trois auteurs Nguyễn Tuân (1988), 'Phở', trong Cảnh sắc và hương vị đất nước, NXB Tác Phâm Mới, Hà Nội, tr. 232. Thạch Lam (2000), 'Quà Hà Nội, Hàng quà rong', trong Hà Nội 36 phố phường , NXB Văn Hóa Thông Tin, Hà Nội, tr.125-127 Thạch Lam (2000), 'Phụ thêm vào phở', trong Hà Nội 36 phố phường, NXB Văn Hóa Thông Tin, Hà Nội, tr.136 Vũ Bằng (2002), 'Phở bò, món quà căn bản', trong Miếng ngon Hà Nội, NXB Văn Học, 2002, p.419-432

Nguyễn, Tôn Nữ Hoàng Mai, (1998), Parlons vietnamien. Langue et culture, Việt Ngũ. Ngôn ngũ Văn hóa. Éditions L'Harmattan. Paris. 
Phong Điệp (2015), Les bols de Phỏ - traduction de Do Thanh Hurinville - Catherine Guy - Minh Ha Lo-Cicero, du vietnamien en français in Impressions d'ExtrêmeOrient $\mathrm{N}^{\circ} 5,2015$, Boire et manger dans les littératures d'Asie. Référence électronique on line: ISSN électronique 2107-027X.

Robert, P. (1989) Le Petit Robert, Dictionnaire, alphabétique et analogique de la langue française. Rédaction dirigée par A. Rey et J. Rey-Debove, LE ROBERT, Paris XI.

Viện Ngôn Ngữ (Institut de Linguistique), Hoàng, P., \& Ali. (1994), Tụ Điển Tiếng Việt Nhà Xuat Ban Khoa Hoc Ha Noi, Trung Tầm Tự Điển Hoc, Hà Nội.

Riegel, M, Pellat J.-C., \& Rioul R. (1996), Grammaire méthodique du français. Paris: PUF Linguistique Nouvelle.

Vũ, Bằng. Phở Bò, Món quà căn bản/La soupe Phở, (2015), La Soupe Phở au bouf, une gourmandise populaire, traduit du vietnamien en français par Minh Ha Lo-Cicero. 